\title{
Variación estacional de las características seminales del bagre rayado Pseudoplatystoma metaense (Telostei, pimelodidae)
}

\author{
Stational variation on seminal characteristics in bagre rayado \\ Pseudoplatystoma metaense (Telostei, pimelodidae)
}

Juan Ramirez-Merlano, ${ }^{1 *}$ M.Sc, Víctor Medina-Robles, ${ }^{1}$ M.Sc, Pablo Cruz-Casallas,${ }^{1}$ Ph.D.

${ }^{1}$ Universidad de los Llanos. Facultad de Ciencias Agropecuarias y Recursos Naturales. Instituto de Acuicultura de la Universidad de los Llanos. Grupo de Investigación sobre Reproducción y Toxicología de Organismos Acuáticos GRITOX. Km 12 vía Puerto López, A.A. 110, Villavicencio, Meta, Colombia. *Correspondencia: Juanantonioramirez.merlano@.gmail.com.

Recibido: Noviembre de 2009; Aceptado: Julio de 2010.

\section{RESUMEN}

Objetivo. Determinar la variación de la calidad, el contenido iónico y la osmolaridad del plasma seminal de Pseudoplatystoma metaense durante la estación reproductiva. Materiales y métodos. Se utilizaron machos sexualmente maduros durante un periodo reproductivo (marzo a agosto). La espermiación fue inducida con Extracto de Hipófisis de Carpa (4 mg/kg). Se evaluó el volumen $(\mathrm{mL})$, movilidad masal (\%), tiempo de activación $(\mathrm{sg})$, espermatocrito (\%); concentración espermática (106 sptz/ $\mu \mathrm{L})$ y viabilidad (\%). También fue determinada la movilidad (\%) y velocidad individual por medio de un Sistema de Análisis Espermático Asistido por Computador (CASA). La osmolaridad (mOsm/Kg) y la concentración de iones $\left(\mathrm{Na}^{+}, \mathrm{Cl}^{+}, \mathrm{K}^{+}\right.$y $\mathrm{Mg}^{2+}$ ) y glucosa fue determinada en plasma seminal obtenido por centrifugación del semen a $14.000 \mathrm{~g}$. La concentración de iones y glucosa fue establecida a través de un sistema de reflectancia. Resultados. El semen de Pseudoplatystoma metaense presentó una movilidad masal mayor al $90 \%$, con el menor

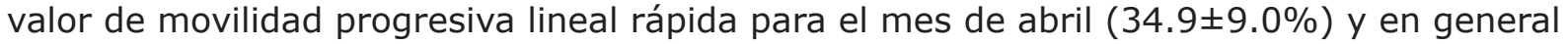
valores de espermatozoides inmóviles menores al 10\%. El plasma seminal mostró una osmolaridad de $259.3 \pm 3.5 \mathrm{mOsm} / \mathrm{Kg}$, con una concentración promedio de glucosa e iones $\mathrm{Na}^{+}, \mathrm{Cl}^{+}, \mathrm{K}^{+}$y Mg${ }^{2+}$ de $142.7 \pm 2.8,118 \pm 2.2,4.8 \pm 0.4$ y $0.33 \pm 0.0 \mathrm{mmol} / \mathrm{L}$, respectivamente, durante la época reproductiva. Conclusiones. La calidad seminal de bagre rayado no presentó variaciones en la estación reproductiva mostrando una alta calidad para los procesos de fecundación.

Palabras clave: Pseudoplatystoma metaense, semen, reprodución. (Fuente: AIMS) 


\section{ABSTRACT}

Objective. To determine the quality variation, the ionic content and the osmolality in Pseudoplatystoma metaense seminal plasma during the reproductive period. Materials and methods. Sexually mature males were used during a reproductive season (march to august). The spermiation was induced with Carp Pituitary Extract $(4 \mathrm{mg} / \mathrm{kg})$. Volume (mL), masal motility (\%), activation time (sc), spermatocrit (\%), sperm concentration (10 $\mathrm{sptz} / \mu \mathrm{L})$ and viability (\%) were evaluated. Also, motility (\%) and individual speed through a Computer-Assisted Semen Analysis (CASA) were determined. The Osmolarity $(\mathrm{mOsm} / \mathrm{kg})$, the ions $\left(\mathrm{Na}^{+}, \mathrm{Cl}^{+}, \mathrm{K}^{+}\right.$ and $\mathrm{Mg}^{2+}$ ) and the glucose concentration were determined in seminal plasma obtained by centrifugation at $14.000 \mathrm{~g}$. The ions and glucose concentration were established through a reflectance system. Results. Sperm showed masal motility greater than $90 \%$, with the

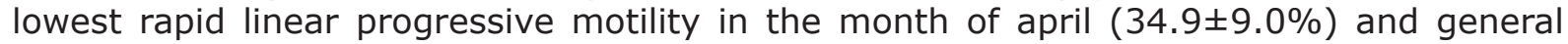
levels of spermatozoa inmotile less than $10 \%$. The seminal plasma showed an osmolarity of $259.3 \pm 3.5 \mathrm{mOsm} / \mathrm{kg}$, with an average concentration of glucose and ions $\mathrm{Na}^{+}, \mathrm{Cl}^{+}, \mathrm{K}^{+}$ and $\mathrm{Mg}^{2+}$ of $142.7 \pm 2.8,118 \pm 2.2,4.8 \pm 0.4$ and $0.33 \pm 0.0 \mathrm{mmol} / \mathrm{L}$, respectively, during the reproductive season. Conclusions. The semen quality of bagre rayado presented no changes during the breeding season, and showed a high quality for the process of fertilization.

Key words: Pseudoplatystoma metaense, sperm, reproduction. (Source: AIMS)

\section{INTRODUCCIÓN}

Los espermatozoides de la mayoría de los peces teleósteos difieren de los mamíferos en aspectos importantes como su inmovilidad en el fluido seminal, inicio de la movilidad inducida por contacto con el agua, corto tiempo de activación y la no presencia de acrosoma $(1,2)$.

La calidad del semen es conferida por la adquisición de la movilidad espermática, sus características bioquímicas, capacidad de ser almacenado por corto tiempo y la resistencia a la crioconservación, así como la capacidad del espermatozoide para fecundar (3).

En peces, variables como el porcentaje de movilidad (movimiento progresivo), duración del movimiento y concentración espermática han sido tradicionalmente utilizadas como patrón de calidad seminal (4). Sin embargo, estas metodologías están basadas en observaciones subjetivas y de alta variabilidad según la precisión del evaluador. Para optimizar y mejorar estas metodologías de evaluación espermática y mostrar valores reales del movimiento y velocidad, varios autores han reportado la utilización de programas computacionales basados en el análisis de imagenes usados en la evaluación de fertilidad humana los cuales han sido adaptados para semen de peces $(5,6)$; dentro de estos programas se encuentra el CASA (Sistema de Análisis Espermático Asistido por Computador) el cual compara los parámetros de movimiento y velocidad de los espermatozoides bajo diferentes condiciones fisiológicas, desempeñando un rol importante para la acuicultura mediante la optimización de las condiciones de manejo y almacenamiento del semen usado para la fecundación de los oocitos (7).

De igual forma, la determinación de las características del semen es necesaria para entender la bioquímica básica que ocurre en la movilidad del espermatozoide y durante la fertilización. El plasma seminal en su composición contiene sustancias de soporte y otras que reflejan las funciones de los espermatozoides y del sistema 
reproductivo, cuyo objetivo es crear un ambiente óptimo para el almacenamiento de los espermatozoides (8). El plasma seminal en muchos peces teleósteos es un producto secretado de los testículos y ductos espermáticos (9). En contraste a otros vertebrados, el fluido seminal es caracterizado por una baja concentración de proteína, conteniendo principalmente iones $\left(\mathrm{Na}^{+}, \mathrm{K}^{+}, \mathrm{Ca}^{+} \mathrm{Mg}^{2+}\right)$ y una baja concentración de otras sustancias orgánicas como el colesterol, glucosa y lípidos (10).

Se han evaluado los cambios estacionales de la calidad espermática en algunas especies de peces determinando el volumen seminal, activación espermática, movilidad, espermatocrito y concentración espermática $(10,11)$. Sin embargo, son pocos los estudios de este tipo que se han realizado en especies con potencial comercial, donde también se encuentran limitaciones en cuanto a la investigación de aspectos de biología y fisiología reproductiva (12), como es el caso del bagre rayado (Pseudoplatystoma metaense) un pimelódido que se encuentra en los principales ríos de Colombia, con un alto potencial para ser utilizado en los procesos de piscicultura, el cual es considerado una especie en riesgo de extinción (13), debido a su captura indiscriminada y al disturbio ocasionado a su hábitat.

Medina-Robles et al (14), reportan un estudio preliminar evaluando el semen de bagre rayado inducido con Extracto de Hipófisis de Carpa (EHC), mostrando un volumen de $9.9 \pm 3.2 \mathrm{~mL}$ con una movilidad masal mayor al $90 \%$, siendo necesario una evaluación más específica de las características seminales de esta especie. Trabajos dirigidos a determinar la variación estacional en las características seminales de Pseudoplatystoma metaense no se encuentran reportados, no obstante, variaciones en la calidad seminal de Brycon amazonicus, un pez también nativo de la región Orinoquia de Colombia, fueron reportados por Cruz-Casallas et al (15).

El objetivo de este estudio fue determinar la variación de la calidad seminal, así como algunos contenidos iónicos y osmolaridad en el plasma seminal de Pseudoplatystoma metaense durante la época reproductiva comprendida entre los meses de marzo y agosto.

\section{MATERIALES Y MÉTODOS}

Localización y descripción del área del estudio. La investigación se llevó a cabo en el Laboratorio de Reproducción de Peces Tropicales y en la Estación Piscícola del Instituto de Acuicultura de la Universidad de los Llanos, localizado a $4 \mathrm{Km}$ de la ciudad de Villavicencio, Meta. El clima se caracteriza por una altura promedio de 420 msnm, temperatura de $25^{\circ} \mathrm{C}$, precipitación pluvial de $4050 \mathrm{~mm}$ y humedad relativa del $75 \%$.

Material biológico. Para todos los ensayos fueron empleados animales sexualmente maduros, provenientes del río Meta, los cuales fueron distribuidos aleatoriamente y mantenidos en estanques en tierra a una densidad de 0.5 animales por $\mathrm{m}^{2}$ y alimentados diariamente con un concentrado del $30 \%$ de proteína bruta. Estos animales fueron mantenidos en cautiverio por un periodo de un año antes de dar inicio a las evaluaciones. Los ejemplares fueron seleccionados de acuerdo con la presencia de semen en la papila urogenital después de un leve masaje cráneo caudal del abdomen. Durante la fase experimental los animales fueron mantenidos en tanques circulares (7000 L) con aireación constante y recambio de agua. Machos diferentes fueron utilizados para cada mes de evaluación.

Inducción hormonal y manejo del semen. La espermiación fue inducida con la aplicación intramuscular de una única inyección de $\mathrm{EHC}(4.0 \mathrm{mg} / \mathrm{Kg}$ de peso corporal, EHC, Stoller Fisheries, USA). El semen fue colectado a las 18 horas postinducción. Previo a la extracción del semen los ejemplares fueron tranquilizados por inmersión en una solución anestésica de 2-fenoxietanol (300 ppm, Sigma Chemical Co., St Louis, MO, USA), durante 5 min. Inmediatamente después de la pérdida del eje de nado, los animales fueron retirados de la solución y secados 
en la región abdominal, con el fin de evitar el contacto del semen con el agua. El semen fue colectado directamente en tubos de vidrio aforados de $15 \mathrm{~mL}$. En todos los casos muestras con presencia de agua, orina, bilis, materia fecal o sangre, fueron descartadas.

Evaluación seminal durante la estación reproductiva. Para evaluar la calidad del semen recolectado después del tratamiento hormonal, inicialmente se registró el volumen $(\mathrm{mL})$ después de la colecta, el cual fue determinado a través de un tubo de ensayo graduado y estéril. Las muestras colectadas fueron mantenidas bajo condiciones de laboratorio $\left(26 \pm 1^{\circ} \mathrm{C}\right)$.

La movilidad masal inicialmente se evaluó de forma subjetiva y fue expresada en porcentaje (\%), para este propósito se activó una alícuota de semen $(20 \mu \mathrm{L})$, colocada en una lámina excavada (1.0-1.2 mm de profundidad, Micro - Slides Premiere, China) bajo microscopio óptico (Zeiss, Alemania, $10 \mathrm{X}$ de magnificación) con $180 \mu \mathrm{L}$ de agua destilada. Posterior a la activación espermática, se determinó el tiempo de activación, hasta la inmovilidad del $90 \%$ de los espermatozoides y fue expresada en segundos. El espermatocrito definido como la relación entre el volumen de células espermáticas empaquetadas y el volumen total del semen, fue expresado en porcentaje (\%) y determinado por la centrifugación (14000 gravedades X 15 min, EBBA 12, Hettich, Tuttlingen, Germany) de una muestra de semen en tubos de hematocrito de $75 \mathrm{~mm}$ de longitud y un diámetro interno de $1.1 \mathrm{~mm}$.

La concentración espermática se determinó dentro de las 8 horas siguientes a la colección del semen por recuento en cámara de Neubauer, previa dilución del semen 1:4000, con solución salina formolada $(0.9 \%$ de $\mathrm{NaCl}$ y formol al $3 \%)$. La cámara se mantuvo en atmósfera húmeda durante al menos 10 min antes del conteo de las células (40X). Para cada muestra, la evaluación se realizó por duplicado y el promedio de las dos lecturas fue utilizado para los análisis subsecuentes.
Para determinar las características morfométricas del espermatozoide de bagre rayado como la longitud de la cabeza y el flagelo, el semen fresco fue diluido en una solución de formaldehido 35\% (4\%) - citrato de sodio [2.9\%, $\mathrm{Na}_{2} \mathrm{H}\left(\mathrm{C}_{3} \mathrm{H}_{5} \mathrm{O}\right.$ $(\mathrm{COO})_{3}$ ] y agua destilada, y registrados por medio del software ACT-2U (NIKON, ACT-2U Imaging Software, Japan), teniendo como punto de referencia o patrón de medida una reglilla $(1000 \mu \mathrm{m})$ y posteriormente calibrada en un microscopio (Nikon E400, Japón).

Los ejemplares fueron seleccionados durante la estación reproductiva comprendida desde el mes de marzo hasta el mes de agosto [marzo $(n=2)$; abril $(n=9)$; mayo $(n=4)$; junio $(n=5)$; julio $(n=7)$; agosto $(n=4)]$ del año 2008. Solo se evaluaron dos animales en marzo, ya que era el inicio de la época reproductiva y no fueron encontrados más animales maduros. El número de animales evaluados por mes se ajusto al número de animales maduros y su cantidad dentro del stock de reproductores mantenidos en el Instituto de Acuicultura de la universidad de los Llanos.

\section{Movilidad y velocidad espermática} individual. Tanto la movilidad como la velocidad espermática individual fueron determinadas a través de un sistema de análisis espermático asistido por computador CASA (Medealab CASA-versión 5.4, Medea AVG, Alemania), adecuando una cámara de Makler (área de $1 \mathrm{~mm}^{2}$, SefiMedical Innstruments, Israel), a un microscopio óptico de contraste de fase (Nikon E400, Japón). Posterior a esto, se colocó una alícuota de semen (c.a. 1 uL) en una cámara de Makler para su evaluación y activada con agua destilada (c.a. 75uL). Para determinar la movilidad individual, fueron observados aproximadamente 200 espermatozoides por campo. Las características seminales evaluadas fueron:

Movilidad progresiva lineal rápida (MPLR). Movilidad progresiva lineal lenta (MPLL). Movilidad local o nado en círculo (MLC). Inmóviles (IMV). Movilidad total espermática individual (MTI). Movilidad circular espermática (MC). 
Velocidad curvilínea (VCL).

Velocidad en línea recta (VLR).

Velocidad promedio de desplazamiento (VPD).

Las movilidades fueron expresadas en porcentaje (\%) y las velocidades en $\mu \mathrm{m} /$ sg. Para la determinación de la movilidad y velocidad individual las evaluaciones fueron realizadas a partir del mes de abril.

\section{Evaluación de la osmolaridad y características bioquímicas del plasma seminal. Fracciones de las muestras seminales obtenidas, fueron centrifugadas a 14000 gravedades (EBBA 12, Hettich, Tuttlingen, Germany) durante 15 min, retirando el sobrenadante (plasma seminal) y almacenándolos en tubos Eppendorf a $-20^{\circ} \mathrm{C}$ para su posterior análisis.}

La osmolaridad del plasma seminal fue determinada tomando un volumen de 10 $\mu \mathrm{L}$ para cada muestra y sometida a un osmómetro (Osmette III $^{\mathrm{TM}}$, Model 5010 Automatic Osmometer, USA), usando un punto de depresión y congelación; para cada evaluación se utilizó agua destilada como solución control. Los valores para esta variable fueron expresados en $\mathrm{mOsm} / \mathrm{Kg}$.

Para determinar las concentraciones de los iones $\mathrm{Na}+, \mathrm{Cl}+, \mathrm{K}+\mathrm{y} \mathrm{Mg}_{2}+(\mathrm{mmo} / \mathrm{L})$, las muestras de plasma seminal fueron incubadas $\left(3 \mathrm{~min}\right.$ a $25^{\circ} \mathrm{C}$ ) y sometidas a un sistema de reflectancia de fibra óptica (VITROSDT60II System Chemistry, USA). Por su parte la glucosa se determinó a través de un analizador químico (R-A 50 Chemistry Analyzer, Alemania), usando una muestra blanco de referencia (Reactive spinreact Glucose TR).

Análisis estadístico. Los valores fueron procesados por medio de análisis descriptivo y expresados como media \pm error estándar de la media (SEM). Para evaluar la variación estacional de las características seminales entre meses, se empleó un análisis de varianza de una vía (ANOVA), seguida de una prueba de Tukey para comparación de medias. Para direccionar el análisis estadístico inicialmente los datos fueron sometidos a una prueba de normalidad (Kolmogorov y Smirnov). Se tomó un valor de $p<0.05$ para considerar diferencias estadísticas. Todos los procedimientos estadísticos fueron realizados empleando el software SAS System versión 8 para Windows (1999-2001 por SAS Institute Inc, Cary, NC. USA).

\section{RESULTADOS}

Evaluación seminal. Los espermatozoides de bagre rayado fueron determinados como uniflagelados, con cabeza en forma cilíndrica (Figura 1), longitud de la cabeza de $1.6 \pm 0.05 \mu \mathrm{m}$ y longitud flagelar de $21 \pm 0.81 \mu \mathrm{m}$.

La estación reproductiva de bagre rayado Pseudoplatystoma $s p$, se presentó desde el mes de marzo hasta el mes de agosto. Las características seminales son mostradas en la tabla 1. El volumen seminal presentó un aumento entre los meses de julio y agosto (8.4 \pm 2.7 y $11.4 \pm 0.7 \mathrm{~mL}$, respectivamente) sin presentar diferencias estadísticas significativas $(p>0.05)$ entre estos, y con una movilidad mayor al $90 \%$ presente en toda la estación reproductiva. Las demás variables mostraron un comportamiento similar $(p>0.05)$. No obstante, se observaron variaciones en el tiempo de Activación (TA), siendo significativamente

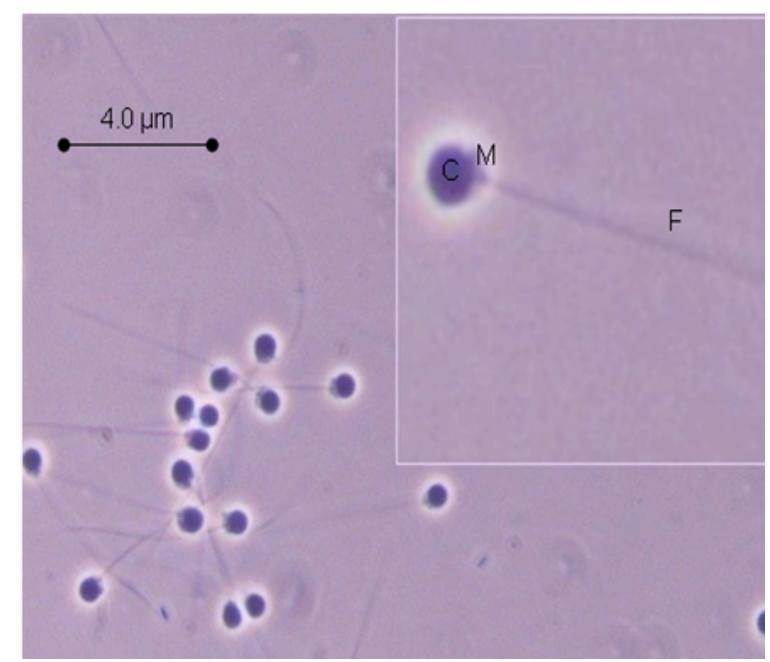

Figura 1. Morfología del espermatozoide de bagre rayado (Pseudoplatystoma metaense), usando un microscopio de contraste de fase (100X). Montaje en fresco. C. cabeza; M. pieza media; F. flagelo. 
Tabla 1. Variación estacional de las características seminales de bagre rayado (Pseudoplatystoma metaense) durante la estación reproductiva. Valores mostrados como media \pm error estándar (SEM). Entre columnas, valores sin superíndices son significativamente iguales $(p>0.05)$

\begin{tabular}{lcccccc}
\hline \multirow{2}{*}{ Variable } & \multicolumn{5}{c}{ Periodo reproductivo } \\
& Marzo $(n=2)$ & Abril $(n=9)$ & Mayo $(n=4)$ & Junio $(n=5)$ & Julio $(n=7)$ & Agosto $(n=4)$ \\
\hline PT $(\mathrm{Kg})$ & $2.1 \pm 0.6$ & $1.6 \pm 0.2$ & $1.3 \pm 0.4$ & $1.3 \pm 0.3$ & $2.1 \pm 0.4$ & $1.6 \pm 0.0$ \\
LT $(\mathrm{cm})$ & $67 \pm 7.0$ & $57.5 \pm 2.6$ & $55 . \pm 5.2$ & $53.2 \pm 5.5$ & $62.2 \pm 2.8$ & $59.3 \pm 0.8$ \\
VOL $(\mathrm{mL})$ & $3 \pm 1.3$ & $6.5 \pm 1.9$ & $4.2 \pm 1.1$ & $6.6 \pm 1.5$ & $8.4 \pm 2.7$ & $11.4 \pm 0.7$ \\
MOV $(\%)$ & $95 \pm 0.0$ & $93.1 \pm 1.8$ & $95 \pm 0.0$ & $95 \pm 0.0$ & $95 \pm 0.0$ & $95 \pm 0.0$ \\
TA $(\mathrm{s})$ & $39.5 \pm 2.5^{\mathrm{b}}$ & $47.3 \pm 1.0^{\text {ab }}$ & $47.3 \pm 2.9^{\text {ab }}$ & $51.1 \pm 2.6^{\text {a }}$ & $51.2 \pm 2.4^{\mathrm{a}}$ & $53 \pm 2.7^{\mathrm{a}}$ \\
ESPT $(\%)$ & $25.0 \pm 13.4$ & $30.0 \pm 4.8$ & $34.8 \pm 8.4$ & $49.2 \pm 3.4$ & $50.6 \pm 5.8$ & $49.8 \pm 2.9$ \\
CE $\left(10^{6} \mathrm{sptz} / \mu \mathrm{L}\right)$ & $30.3 \pm 1.7$ & $28.8 \pm 6.7$ & $44.2 \pm 10.5$ & $35.6 \pm 3.3$ & $38.9 \pm 5.5$ & $31.8 \pm 2.4$ \\
VIAB $(\%)$ & $95.5 \pm 3.4$ & $95.9 \pm 0.9$ & $95.9 \pm 0.9$ & $96.6 \pm 1.1$ & $95.7 \pm 0.4$ & $96.3 \pm 0.8$ \\
\hline
\end{tabular}

PT: peso total; LT: longitud total; VOL: volumen seminal; MOV: movilidad seminal; TA: tiempo de activación espermática; ESPT: espermatocrito; CE: concentración espermática por recuento celular; VIAB: viabilidad. a,b Entre columnas, valores con letras diferentes presentan diferencias significativas $(p<0.05)$.

menor en el mes de marzo (39.5 $\pm 2.5 \mathrm{~s})$, cuando comparado con los tres últimos meses de evaluación (junio a agosto).

\section{Movilidad y velocidad espermática individual}

Movilidad progresiva lineal rápida (MPLR) y Movilidad progresiva lineal lenta (MPLL). El comportamiento de la MPLR para semen de bagre rayado (Pseudoplatystoma sp) durante la estación reproductiva, se observa en la figura 2 . La MPLRal ser evaluada en el mes abril presentó el menor porcentaje (34.94 $9.02 \%)$, en contraste a los meses de mayo y julio con valores de $61.01 \pm 8.26$ y $57.87 \pm 9.02 \%$, respectivamente, sin mostrar diferencias significativas durante la estación reproductiva $(p>0.05)$. Por su parte la MPLL, presentó variaciones en los meses de mayo (26.50 $5.85 \%$ ) $y$ julio (28.46士5.72\%), siendo estos los de menor porcentaje de movilidad (Figura 2), con respecto a los demás meses de evaluación sin mostrar diferencias estadísticas $(p>0.05)$.

Movilidad local o nado en circulo (MLC) y espermatozoides inmóviles (IMV). La evaluación de la movilidad local o nado

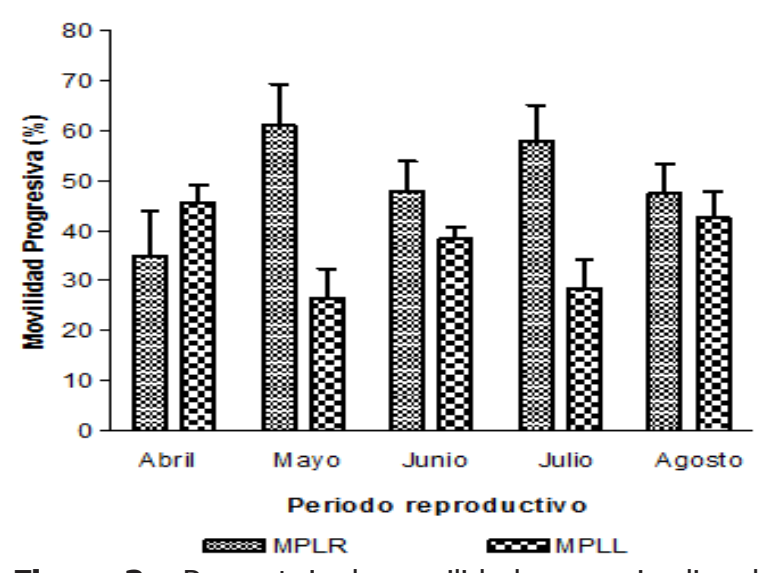

Figura 2. Porcentaje de movilidad progresiva lineal rápida (MPLR) y movilidad progresiva lineal lenta (MPLL) para semen fresco de bagre rayado (Pseudoplatystoma metaense). Los valores son expresados como media \pm error estándar (SEM). $(p>0.05)$. abril $(n=4)$; mayo $(n=4)$; junio $(n=4)$; julio $(n=5)$; agosto $(n=5)$.

en círculo se observa en la figura 3 , con un porcentaje mayor de MLC en el mes de abril $(13.78 \pm 6.80 \%)$, sin mostrar variaciones con los valores determinados en los meses de julio y agosto ( $8.3 \pm 3.9$ y $7.5 \pm 2.2 \%$, respectivamente) durante la estación reproductiva. Al ser comparado este parámetro entre los meses de evaluación no se observó diferencias estadísticas significativas $(p>0.05)$. El semen de bagre 


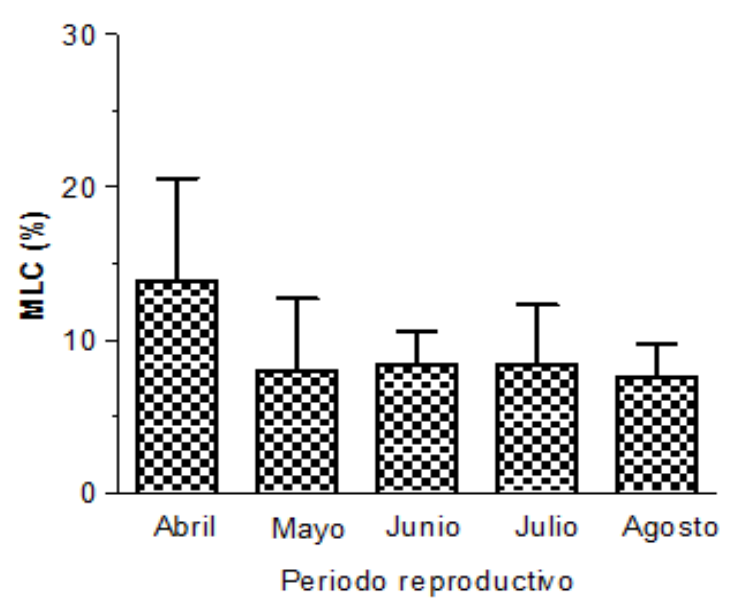

Figura 3. Porcentaje de movilidad local o nado en circulo (MLC) para semen fresco de bagre rayado (Pseudoplatystoma metaense). Los valores son expresados como media \pm error estándar (SEM). ( $p>0.05)$. Abril $(n=4)$; Mayo $(n=4)$; Junio $(n=4)$; Julio $(n=5)$; Agosto $(n=5)$.

rayado mostró valores mínimos $(2.4 \pm 1.3 \%)$ de espermatozoides inmóviles (IMV) en el mes de agosto, observándose el valor más alto en el mes de abril $(5.82 \pm 2.46 \%)$, sin mostrar diferencias estadística con respecto a los otros meses de evaluación $(p>0.05)$ (Figura 4). Se pudo observar que estos dos parámetros de movimiento presentaron similitud durante la estación reproductiva.

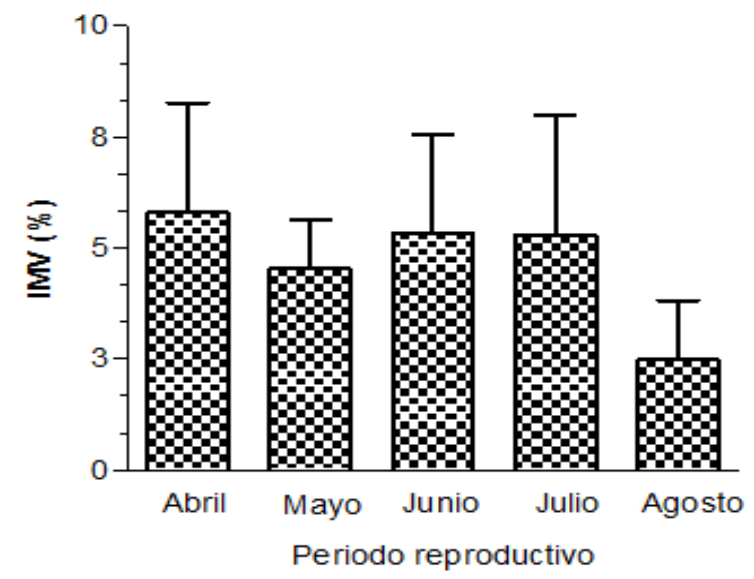

Figura 4. Porcentaje de espermatozoides inmóviles (IMV) para semen fresco de bagre rayado (Pseudoplatystoma metaense). Los valores son expresados como media \pm error estándar (SEM). ( $p>0.05)$. Abril $(n=4)$; Mayo $(n=4)$; Junio $(n=4)$; Julio $(n=5)$; Agosto $(n=5)$.
Movilidad total individual (MTI) y movilidad total circular espermática (MTC). La evaluación de la MTI por medio del CASA (Figura 5), mostró un semen con una movilidad superior al $80 \%$ durante la estación reproductiva, sin diferencias significativas entre los meses evaluados $(p>0.05)$. Por su parte, la MTC (Figura 6) solo fue observada en los meses de junio y julio $(0.9 \pm 0.9$ y $0.3 \pm 0.3 \%$, respectivamente).

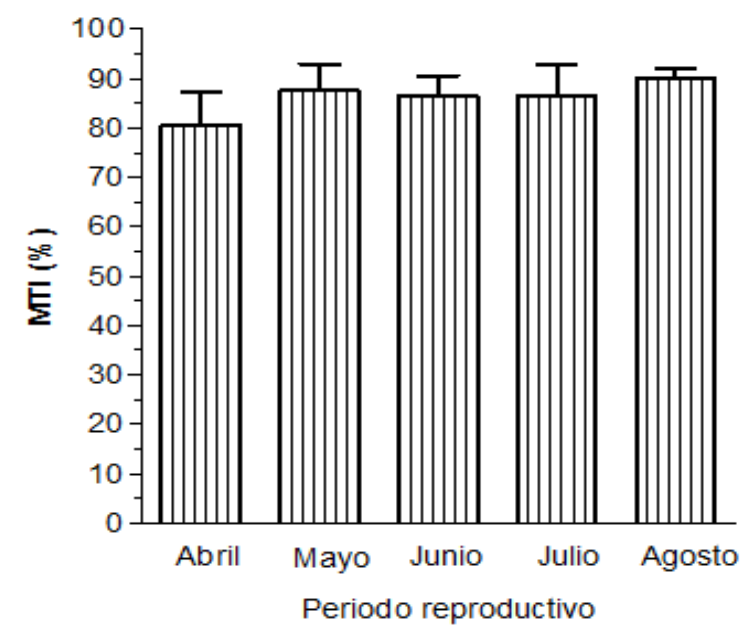

Figura 5. Porcentaje de movilidad total individual (MTI) para semen fresco de bagre rayado (Pseudoplatystoma metaense). Los valores son expresados como media \pm error estándar (SEM). ( $p>0.05)$. Abril $(n=4)$; Mayo $(n=4)$; Junio $(n=4)$; Julio $(n=5)$; Agosto $(n=5)$.

Velocidad en línea recta (VLR),
velocidad curvilínea (VCL) y velocidad
promedio de desplazamiento (VPD). La velocidad en línea recta (VLR) presentó valores superiores para los meses de mayo y julio $(43.7 \pm 6.3$ y $41.6 \pm 3.9$ $\mu \mathrm{m} / \mathrm{s}$, respectivamente), mientras que el valor mínimo se observó en el mes de abril $(27.3 \pm 9.4 \mu \mathrm{m} / \mathrm{s})$ sin diferencias significativas $(p>0.05)$ al compararse con los demás meses evaluados (Figura 7). Otro parámetro de velocidad como es la VCL, mostró variación estacional, siendo mayor en el mes de Mayo $(51.2 \pm 8.4 \mu \mathrm{m} / \mathrm{s})$ y menor en el mes de Abril (34.85 \pm 10.3 $\mu \mathrm{m} / \mathrm{s}$ ), sin embargo, no se presentó diferencias significativas en los meses evaluados ( $p>0.05)$. De igual forma la VPD mostró una tendencia similar con respecto 


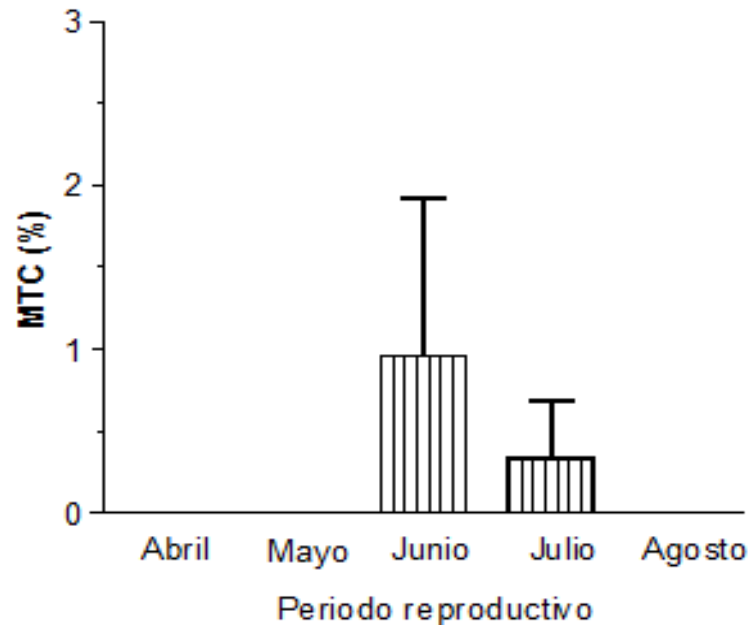

Figura 6. Porcentaje de movilidad total circular (MTC) para semen fresco de bagre rayado (Pseudoplatystoma metaense). Los valores son expresados como media \pm error estándar (SEM). ( $p>0.05)$. abril $(n=4) ;$ mayo $(n=4)$; junio $(n=4)$; julio $(n=5)$; agosto $(n=5)$.

a las anteriores velocidades mencionadas, con un valor mínimo de velocidad de $29.7 \pm 9.9 \mu \mathrm{m} / \mathrm{s}$ registrado en el mes de abril, sin diferencias significativas con respecto a los otros meses de evaluación $(p>0.05)$.

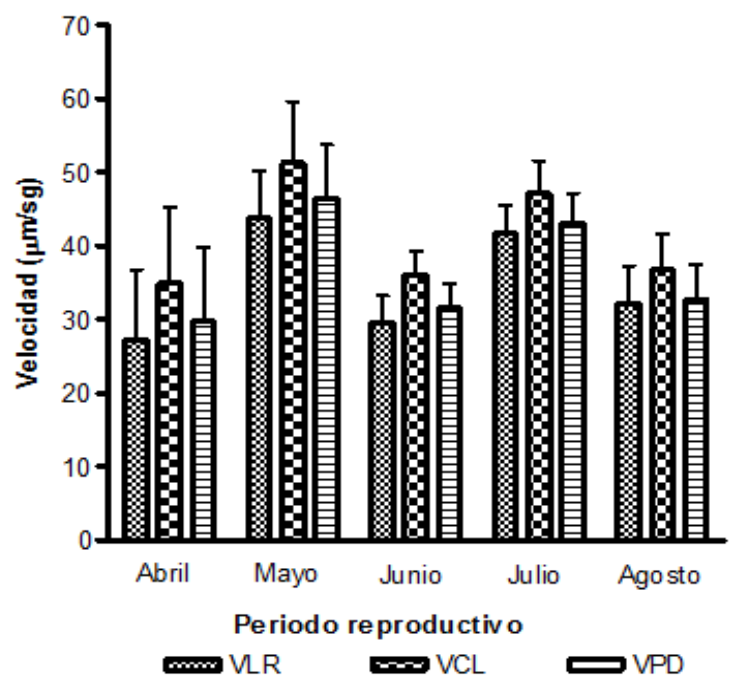

Figura 7. Velocidades espermáticas: en línea recta (VLR), curvilínea (VCL), velocidad promedio de desplazamiento (VPD) para semen fresco de bagre rayado (Pseudoplatystoma metaense). Los valores son expresados como media \pm error estándar (SEM). ( $p>0.05)$. abril $(n=4) ;$ mayo $(n=4) ;$ junio $(n=4)$; julio $(n=5)$; agosto $(n=5)$.

\section{Osmolaridad y características bioquímicas} del plasma seminal. El análisis de la composición del plasma seminal para bagre rayado, no presentó cambios significativos durante la estación reproductiva, los

Tabla 2. Osmolaridad y concentración de glucosa e iones $\mathrm{Na}^{+}, \mathrm{Cl}^{+}, \mathrm{K}^{+}$y $\mathrm{Mg}^{2+}$, en el plasma seminal de bagre rayado (Pseudoplatystoma metaense) durante la estación reproductiva. Valores mostrados como media \pm error estándar (SEM). $(p>0.05)$.

\begin{tabular}{lcccccc}
\hline \multicolumn{1}{c}{ Variable } & $\begin{array}{c}\text { Marzo } \\
(\mathbf{n = 2})\end{array}$ & $\begin{array}{c}\text { Abril } \\
(\mathbf{n = 9})\end{array}$ & $\begin{array}{c}\text { Mayo } \\
(\mathbf{n = 4})\end{array}$ & $\begin{array}{c}\text { Junio } \\
(\mathbf{n = 5})\end{array}$ & $\begin{array}{c}\text { Julio } \\
(\mathbf{n}=\mathbf{7})\end{array}$ & $\begin{array}{c}\text { Agosto } \\
(\mathbf{n}=\mathbf{4})\end{array}$ \\
\hline OSM $(\mathrm{mOsm} / \mathrm{Kg})$ & $263.8 \pm 10.1$ & $260.8 \pm 11.98$ & $269.6 \pm 6.43$ & $225.8 \pm 19.55$ & $270.5 \pm 4.7$ & $268.8 \pm 11.0$ \\
$\mathrm{Na}+(\mathrm{mmol} / \mathrm{L})$ & $152 \pm 14.0$ & $136.4 \pm 5.2$ & $147 \pm 2.6$ & $137.3 \pm 12.8$ & $145.7 \pm 3.9$ & $147.2 \pm 7.4$ \\
$\mathrm{Cl}^{+}(\mathrm{mmol} / \mathrm{L})$ & $121.5 \pm 6.5$ & $116.2 \pm 4.2$ & $119.6 \pm 1.8$ & $111.6 \pm 9.5$ & $122.5 \pm 2.5$ & $120.2 \pm 7.1$ \\
$\mathrm{~K}^{+}(\mathrm{mmol} / \mathrm{L})$ & $2.5 \pm 1.0$ & $4.9 \pm 0.9$ & $3.9 \pm 0.2$ & $3.4 \pm 1.2$ & $6.4 \pm 0.3$ & $6.1 \pm 0.9$ \\
$\mathrm{Mg}^{2+}(\mathrm{mmol} / \mathrm{L})$ & $0.4 \pm 0.2$ & $0.3 \pm 0.07$ & $0.2 \pm 0.0$ & $0.2 \pm 0.0$ & $0.3 \pm 0.0$ & $0.2 \pm 0.0$ \\
$\begin{array}{l}\mathrm{Glucosa} \\
(\mathrm{mmol} / \mathrm{L})\end{array}$ & $0.2 \pm 0.1$ & $0.4 \pm 0.07$ & $0.7 \pm 0.4$ & $0.2 \pm 0.0$ & $0.5 \pm 0.1$ & $0.1 \pm 0.0$ \\
\hline
\end{tabular}

OSM: osmolaridad 
valores son observados en la tabla 2. La Osmolaridad mostró un comportamiento similar durante los meses evaluados, sin embargo, se observaron valores bajos en el mes de junio con un valor promedio de $225.8 \pm 19.5 \mathrm{mOsm} / \mathrm{Kg}$, sin mostrar diferencia significativa con respecto a los demás meses evaluados $(p>0.05)$. Las concentraciones de iones de $\mathrm{Cl}^{+}$y $\mathrm{K}^{+}$mostraron los valores más altos para el mes de julio (122.5 \pm 2.5 y $6.4 \pm 0.3 \mathrm{mmol} / \mathrm{L}$, respectivamente). Por su parte, la concentración de glucosa no presentó diferencias significativas durante la estación reproductiva evaluada $(p>0.05)$, presentándose una tendencia a la disminución al final de la época reproductiva reproductiva con un valor de $0.1 \pm 0.0 \mathrm{mmol} / \mathrm{L}$.

\section{DISCUSIÓN}

Uno de los prerrequisitos para una acuicultura sostenible es la capacidad de control de los procesos reproductivos de los peces en cautiverio con el fin de adquirir una alta calidad de sus gametos (16). En todos los cultivos de peces la manipulación hormonal es practicada comúnmente para incrementar la espermiación (17). En el presente trabajo la variación durante la estación reproductiva del semen de bagre rayado fue evaluada mediante inducción hormonal. Rurangwa et al (10) reportan que los tratamientos hormonales pueden producir una variación sobre los parámetros fisiológicos del semen.

Los resultados de calidad seminal de bagre rayado en el presente trabajo mostraron un volumen seminal con un promedio mínimo y máximo de 3 y $11 \mathrm{~mL}$, respectivamente $(p>0.05)$, siendo muy variable entre individuos, estos valores se encuentran dentro de lo reportado por MedinaRobles et al (14) para la misma especie

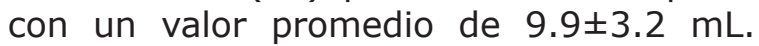
En contraste, Mansour et al (5), reportan un volumen inferior de $1.7 \pm 0.2 \mathrm{~mL}$ para Clarias gariepinus. En especies como el yaque (Leiarius marmoratus) variables como volumen seminal, movilidad y TA $(3.0 \pm 0.9 \mathrm{~mL} ; 76.1 \pm 5.4 \%$ y $43.7 \mathrm{sg}$, respectivamente) (18), se encuentran por debajo a lo reportado en este trabajo, siendo los tratamientos hormonales una alternativa para obtener muestras seminales de volúmenes aceptables, especialmente para especies que presentan dificultad para la expulsión de semen por medio de una leve presión cráneo caudal (19).

El tiempo de activación fue alto ( $53 \pm 2.7$ $\mathrm{sg})$, al final de la estación reproductiva $(p<0.05)$, cercano a lo reportado para Pangasius bocourti (20). Comparados con las especies marinas (mayor a $2 \mathrm{~min}$ ) los anteriores valores son menores (2), atribuibles posiblemente al control de la presión osmótica. En peces de agua dulce, el agua tiene una baja osmolaridad en comparación con el plasma seminal, ocasionando un choque hiposmótico y un rápido deterioro del espermatozoide conllevando a movimientos de baja calidad después de 30 s (20).

En la producción de peces comerciales, la evaluación de la calidad del semen es importante para la eficiencia en la propagación artificial y en otros procesos como la crioconservación, acorde con las estrategias reproductivas de la especie. Los parámetros más comunmente empleados en el estudio de la biología del espermatozoide son la estructura y parámetros de la movilidad $(2,18)$. De igual forma, son escasos los estudios donde incorporen la evaluación de la movilidad y velocidad individual de espermatozoides de peces, por medio de análisis espermático asistido por computador.

En el presente trabajo la MPLR presentó variaciones no significativas ( $p>0.05)$. En la fase intermedia de la estación reproductiva esta velocidad presentó el valor más alto similar a lo reportado por Medina-Robles et al (6) al evaluar el semen de yaque (Leiarius marmuratus) inducido con Ovaprim $\AA$ en dosis única $(0.25 \mathrm{~mL})$ con un valor promedio de $(60.1 \pm 13.1 \%)$ y un porcentaje de $2.9 \pm 2.2 \%$ de espermatozoides inmóviles, siendo menor a lo reportando en este estudio. Por su parte la MPLL, presentó variaciones no significativas durante la estación reproductiva $(p>0.05)$. Kwantong y Bart (21), evaluaron la movilidad para 
Pangasius larnaudii con una movilidad total de $88.30 \pm 10 \%$ utilizando LHRHa y domperidona $(10 \mu \mathrm{g} / \mathrm{Kg}-5 \mathrm{~m} / \mathrm{Kg}$, respectivamente) similar a lo reportando en este estudio y mayor a lo determinado en semen intratesticular de Clarias gariepinus con un valor de $74.6 \pm 6.1 \%(22)$.

En peces, la trayectoria del espermatozoide es generalmente más curva que en mamíferos y puede moverse tridimensional en el medio acuoso (7), sin embargo, Rurangwa et al (23) mostraron valores de movilidad total de $98.5 \%$ y una VLR y VCL de $38 \mu \mathrm{m} / \mathrm{s}$ y $58 \mu \mathrm{m} / \mathrm{s}$, respectivamente, para esta misma especie. Estas velocidades son los parámetros más usados puesto que la velocidad del espermatozoide de los peces disminuye rápidamente con el tiempo, donde la duración de movimientos progresivos tiene una alta influencia sobre la habilidad del espermatozoide para entrar al oocito (7). Los valores de VLR son mayores en comparación con el bagre africano (Clarias gariepinus) anteriormente mencionado, mientras que la $\mathrm{VCL}$ es cercana a lo reportado por Rurangwa et al (23) en la misma especie.

En yaque los valores de velocidad reportados son menores, con una VLR de $24.8 \pm 3.3$ y $V C L$ de $28.3 \pm 2.8 \mu \mathrm{m} / \mathrm{s}$ (6), mientras que Warncke y Pluta (24), reportan una VPD $(40 \mu \mathrm{m} / \mathrm{s})$ para Cyprinus carpio, cercano a lo reportado para bagre rayado en el presente trabajo.

Los valores de movimiento en nado circular fueron inferiores al 15\%, siendo un semen caracterizado por una movilidad con un nado progresivo y un bajo porcentaje de MTC. La evaluación de la movilidad por medio del CASA durante la estación reproductiva muestra al semen de bagre rayado de alta calidad, con una viabilidad para los procesos de reproducción artificial y crioconservación.

En cuanto a las concentraciones de iones, para el presente estudio, estas son comparadas con lo reportado para Clarias macrocephalus (25); Petromyzon marinus (26); Tinca tinca (27); Acipenser persicus (28) y Brycon amazonicus (11), observándose que la concentración de $\mathrm{Na}^{+}$es cercana a los rangos reportados para Clarias macrocephalus (164.4 0.6 $\mathrm{mmol} / \mathrm{L})$ y Petromyzon marinus $(105.3 \pm 12.8 \mathrm{mmol} / \mathrm{L})$, mientras que en especies como Tinca tinca (18.4 1.3 $\mathrm{mmol} / \mathrm{L})$ y Brycon amazonicus (81.4 45.2 $\mathrm{mmol} / \mathrm{L}$ ) se reportan valores por debajo de lo determinado en este estudio. Por su parte, los valores de $\mathrm{Cl}^{+}$son más altos al ser comparados con las especies en mención. El $\mathrm{K}^{+}$fue menor $(6.17 \mathrm{mmol} / \mathrm{L})$ a lo mostrado por Clarias macrocephalus $(17.8 \pm 01 \mathrm{mmol} / \mathrm{L})$ y las concentraciones de $\mathrm{Mg}^{2+}$ determinadas se encuentran entre los rangos reportados para estas especies como Tinca tinca y Acipenser persicus (0.45 0.1 y $0.520 .03 \mathrm{mmol} / \mathrm{L}$, respectivamente).

Las concentraciones de $\mathrm{Na}^{+} \mathrm{Cl}^{+}, \mathrm{K}^{+}$y $\mathrm{Mg}^{2+}$ no mostraron variaciones significativas durante los meses evaluados de la estación reproductiva. Sin embargo, Alavi y Cosson (2), reportan que puede presentarse una variación en la composición iónica durante la estación reproductiva, con una predominancia en los niveles de $\mathrm{Na}^{+}$ y $\mathrm{Cl}^{+}$en el plasma seminal. Por su parte, la glucosa fue inferior a lo reportado en

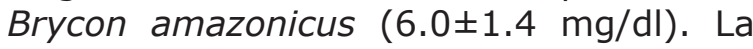
osmolaridad promedio del plasma seminal de bagre rayado para los meses de marzo a agosto, son similares a lo mostrado en Clarias macrocephalus $269.0 \pm 6.4 \mathrm{mmol} / \mathrm{L}$ (24), y menores en comparación con Brycon amazonicus (11), Gadus morhua (29) y Esox lucios L (30) (314.1, 368.2 \pm 8.9 , $283 \pm 33 \mathrm{mOsm} / \mathrm{Kg}$, respectivamente).

Finalmente, la evaluación de la calidad seminal es importante para los procesos de obtención de gametos viables y en procura de conformar un plantel con una viabilidad para los procesos de reproducción y congelación seminal, así como procesos de mejoramiento genético.

En conclusión, la calidad seminal de bagre rayado fue alta durante la estación reproductiva con variaciones no significativas, cuya información constituye el primer reporte de una 
evaluación global en esta especie, proyectando al semen de bagre rayado (Pseudoplatystoma metaense) con posibilidad de ser utilizado para fines de reproducción artificial en todos los meses de la estación reproductiva.

\section{Agradecimientos}

Al Centro Internacional de Agricultura Tropical - Ministerio de Agricultura y Desarrollo Rural proyecto No. 015-1/06 CIAT - Universidad de los Llanos, así como al Instituto de Investigaciones de la Orinoquia Colombiana IIOC por su apoyo financiero y al Instituto de Acuicultura de los Llanos por el apoyo logístico.

\section{REFERENCIAS}

1. Yu S, Kojima N, Hakomori SI, Kudo S, Inoue $S$, Inoue $Y$. Binding of rainbow trout sperm to egg is mediated by strong carbohydrate-tocarbohydrate interaction between (KDN) GM3 (deaminated neuraminyl ganglioside) and Gg3-like epitope. Proc Natl Acad Sci USA 2002; 99:2854-2859.

2. Alavi SMH, Cosson J. Sperm motility in fishes: (I) Effects of temperature and $\mathrm{pH}$ : a review. Cell Biol Int 2005; 29: 101-110.

3. Coward $\mathrm{K}$, Bromage NR, Hibbitt $\mathrm{O}$, Parrngton J. Gamete physiology, fertilization and egg activation in teleost fish. Rev Fish Biol Fisher 2002; 12:33-58.

4. Cruz-Casallas PE, Pardo-Carrasco SP, Arias-Castellanos JA, LomboCastellanos PE, Lombo-Rodriguez DA, Pardo-Mariño JE. Cryopreservation of yamú Brycon siebenthalae milt. J World Aquac Soc 2004; 35: 529-535.

5. Mansour N, Ramoun A, Lahnsteiner F. Quality of testicular semen of the African catfish Clarias gariepinus (Burchell, 1822) and its relationship with fertilization and hatching success. Aquacult Res 2005; 36:1422-1428.
6. Medina-Robles VM, Mira-López T, CruzCasallas PE. Efectos de diferentes inductores hormonales sobre la movilidad y velocidad espermática del yaque (Leiarius marmuratus). En: memorias IV Congreso Colombiano de acuicultura. Rev Colomb Cienc Pecu 2008; 21:455-522.

7. Kime DE, Van Looka KJW, McAllister BG, Huyskensb G, Rurangwa E, Ollevier F. Computer-assisted sperm analysis (CASA) as a tool for monitoring sperm quality in fish. Comp Biochem Phys 2001; 130: 425-433.

8. Ciereszco A. Chemical composition of seminal plasma and its physiological relationship with sperm motility, fertilizing capacity and cryopreservation success, In: Hadi, S.M., Cosson, J., Coward, K., Rafiee, G. (Eds.). Fish Spermatology 2007; 281-316.

9. Lahnsteiner F. Morphology, fine structure, biochemistry, and fuction of the spermatic ducts in marine fish. Tissue Cell 2003; 35:363-373.

10. Rurangwa $E$, Kime DE, Ollevier F, Nash JP. The measurement of sperm motility and factors affecting sperm quality in cultured fish. Aquaculture 2004; 234:1-28. 
11. Cruz-Casallas PE, Medina-Robles VM, Velasco-Santamaría YM. Seasonal variation of sperm quality and the relationship between spermatocrit and sperm concentration in yamú Brycon amazonicus. N Am J Aquacult 2007; 69:259-165.

12. Guerrero ACE. Treinamento alimentar de pintado Pseudoplatystoma coruscans (Agassiz, 1829): Sobrevivência, crescimento e aspectos econômicos. [tesis de maestría]. Jaboticabal, Brazil: São Paulo Uni; 2003.

13. Pinzón-Arciniegas SM, MojicaRodríguez JE, Cruz-Casallas PE. Ensayos preliminares sobre crioconservación de semen de bagre rayado (Pseudoplatystoma fasciatum Linnaeus, 1766). Orinoquía 2005; $9(2): 28-37$.

14. Medina-Robles VM, Guarnizo-Pineda M, Ramírez-Merlano JA, Otero-Paternina AM, Mira T, Pacheco-Murillo R, VelascoSantamaría YM, Cruz-Casallas PE. Caracterización y ensayos preliminares de crioconservación seminal de bagre rayado (Pseudoplatystoma fasciatum - Linnaeus, 1766). En memorias XIII Jornada de Acuicultura. Villavicencio: Universidad de los Llanos: 2007.

15. Cruz-Casallas PE, Medina-Robles VM, Velasco-Santamaría. Seasonal Variation of Sperm Quality and the Relationship between Spermatocrit and Sperm Concentration in Yamu' Brycon amazonicus. N Am J Aquacult 2007; 69:159-165.

16. Duarte M, Marbá N, Holmer M. Rapid domestication of marine species. Science 2007; 16:382-383.

17. Mylonas CC, Fostier A, Zanuy S. Broodstock management and hormonal manipulations of fish reproduction. Gen Comp Endocrinol 2010; 165:516-534.
18. Mira T, Murillo-Pacheco $R$, RamirezMerlano JA, Otero-Paternina AM, Berruecos-Zapata BE, Medina-Robles VM, Cruz-Casallas PE. Ensayos preliminares de reproducción inducida de yaque Leiarius marmuratus con extracto de hipófisis de carpa. En: memorias IV Congreso Colombiano de acuicultura. Rev Colomb Cienc Pecu 2008; 21: 455-522.

19. Viveiros ATM, Godinho HP. Sperm quality and cryopreservation of Brazilian freshwater fish species: a review. Fish Physiol Biochem 2008; 137-150.

20. Cacot $P$, Eeckhourte $P$, Muon DT, Trieu $N V$, Legengdre $M$, Mariojouls, $C$, et al. Induced spermiation and milt management in Pangasius bocourti (Sauvage, 1880). Aquaulture 2003; 215: 67-77.

21. Kwantong S, Bart AN. Cryopreservation of black ear catfish, Pangasius larnaudii, (Bacourt) sperm. Aquacult Res 2006; 37: 955-957.

22. Mansour N, Lahnsteiner $F$, Berger B. Metabolism of intratesticular spermatozoa of a tropical teleost fish (Clarias gariepinus). Comp Biochem Phys part B 2003; 135: 285-296.

23. Rurangwa E, Volckaert FAM, Huyskens IG, Kime IDE, Ollevier, F. Quality control of refrigerated and cryopreserved semen using computerassisted sperm analysis (casa), viable staining and standardized fertilization in african catfish (Clarias gariepinus). Theriogenology 2001; 55: 751-769.

24. Wamecke D, Pluta HJ. Motility and fertilizing capacity of frezen/thawed common carp (Cyprinus carpio L) sperm using dimetil-acetamida as the mam cryoprotectant. Aquaculture 2003; 215: 167-185. 
25. Tan-Fermin JD, Miura T, Adachi $S$, Yamauchi K. Seminal plasma composition, sperm motility, and milt dilution Asian catfish Clarias macrocephalus (Gunther). Aquaculture 1999; 171: 323-338.

26. Ciereszko A, Dabrowski K, Toht GP, Christ SA, Glogowski J. Factors affecting motility characteristics and fertilizing ability of sea Lamprey spermatozoa. Trans Am Fish Soc 2002; 131: 193-202.

27. Linhart O, Rodina M, Bastl J, Cosson J. Urinary bladder, ionic composition of seminal fluid and urine with characterization of sperm motility in tench (Tinca tinca). J Appl Ichthyol 2003; 19: 177-81.

28. Alavi $\mathrm{SMH}$, Cosson J, Karami M, Hossein A, Amiri BM. Chemical composition and osmolality of seminal fluid of Acipenser persicus; their physiological relationship with sperm motility. Aquacult Res 2004; 35: 1238-1243.
29. Suquet M, Rouxel C, Cosson J, Severe A, Quemener L, Fauvel C. Changes in atlantic cod (Gadus morhua) sperm quality with time, In: Hendry, C.I., Van Stappen, G., Wile, M., Sorgeloss, P. (Eds.). Larvi 2005, Eur. Aquac. Soc., sp. Publication 2005. p. 503-505.

30. Alavi SMH, Rodina M, Viveiros ATM, Cosson J, Gela D, Boryshpolets, S, et al. Effects of osmolality on sperm morfphology, motility and flagelar wave parameters in Northern pike (Esox lucius L.). Theriogenology 2009; 72: 32-43. 